\title{
Selection of thrombolytic therapy for individual patients: Development of a clinical model
}

\author{
Robert M. Califf, MD, ${ }^{a}$ Lynn H. Woodlief, MS, ${ }^{a}$ Frank E. Harrell Jr., PhD, ${ }^{b}$ Kerry L. Lee, PhD, ${ }^{\text {b }}$ \\ Harvey D. White, MB, DSc, ${ }^{\mathrm{c}}$ Alan Guerci, MD, ${ }^{\mathrm{d}}$ Gabriel I. Barbash, MD, MPH, ${ }^{\mathrm{e}}$ R. John Simes, MD, ${ }^{f}$ \\ W. Douglas D. Weaver, MD, ${ }^{\mathrm{g}}$ Maarten L. Simoons, MD, ${ }^{\mathrm{h}}$ and Eric J. Topol, MD, ${ }^{\mathrm{i}}$ for the GUSTO-I \\ Investigators Durham, N.C.; Auckland, New Zealand; Roslyn, N.Y.; Tel Aviv, Israel; Sydney, Australia; \\ Seattle, Wash.; and Rotterdam, The Netherlands
}

\begin{abstract}
We developed a logistic regression model with data from the GUSTO-I trial to predict mortality rate differences in individual patients who received accelerated tissue plasminogen activator (TPA) versus streptokinase treatment for acute myocardial infarction. A nomogram was developed from a reduced version of this model that approximated the underlying risk of patients treated with streptokinase, and thus the benefit of TPA. The 30-day mortality rate with accelerated TPA was 0.063 versus 0.073 with streptokinase and subcutaneously administered heparin and 0.074 with streptokinase and intravenously administered heparin. No baseline patient characteristics were significantly associated with a different relative effect of TPA. Older patients and those with anterior infarction, higher Killip classification (except Killip class IV), lower blood pressure, and increased heart rate had the greatest absolute benefit with accelerated TPA. Patients with acute myocardial infarction who had more high-risk characteristics derived a greater absolute benefit from treatment with accelerated TPA versus streptokinase. (Am Heart J 1997;133:630-9.)
\end{abstract}

The clinician treating patients with an acute illness must rapidly make therapeutic decisions on the basis of a brief encounter with the patient. The randomized clinical trial provides a powerful means of

From the Departments of a Medicine (Cardiology) and ${ }^{b}$ Community and Family Medicine (Biometry), Duke University Medical Center, Durham, N.C.; 'Cardiology Department, Green Lane Hospital, Auckland, New Zealand; dSt. Francis Hospital, Roslyn, N.Y.; eDepartment of Medicine, Tel-Aviv-Elias Sourasky Medical Center, Tel Aviv, Israel; fNMHRC Clinical Trials Centre, University of Sydney, Australia; 'DDepartment of Medicine (Cardiology), University of Washington, Seattle, Wash.; ${ }^{\text {hThorax- }}$ center, Rotterdam, The Netherlands; and the iDepartment of Cardiology, Cleveland Clinic Foundation, Cleveland, Ohio.

Supported by grants from Bayer (New York, N.Y.), CIBA-Corning (Medfield, Mass.), Genentech (South San Francisco, Calif.), ICI Pharmaceuticals (Wilmington, Del.), and Sanofi Pharmaceuticals (Paris, France).

Reprint requests: Robert M. Califf, MD, Division of Cardiology, Department of Medicine, Box 31123, Duke University Medical Center, Durham, NC 27710 .

E-mail: calif001@mc.duke.edu

Copyright (c) 1997 by Mosby Year Book, Inc.

$0002-8703 / 97 / \$ 5.00+0 \quad \mathbf{4} / \mathbf{1} / \mathbf{8 1 2 1 6}$ determining the average differences among treatments in a population of patients. However, the clinician also intuitively knows that when one treatment is found to be superior to another in a clinical trial, all patients in a given population will not likely derive the same degree of absolute benefit. Having a rational basis for quantifying the expected degree of treatment benefit for the individual patient would be desirable, especially when the more effective treatment is also more expensive.

In describing and testing a treatment effect when the outcome is a discrete event, basing an outcomes model on a measure of relative effect, such as an odds ratio, is customary. The major reason for this approach to quantifying treatment effect is the fact that odds ratios can be constant over a broad range of baseline patient risks or severity of illness. When the relative benefit is constant, the absolute benefit necessarily varies according to baseline risk. For example, a patient with a probability of death of 0.001 can receive a maximum absolute benefit of 0.001 , whereas a patient with a probability of 0.5 can receive an absolute benefit of up to 0.5 .

In the Global Utilization of Streptokinase and t-PA for Occluded Coronary Arteries (GUSTO-I) trial, ${ }^{1}$ administration of accelerated tissue plasminogen activator (TPA) and intravenous heparin resulted in a survival benefit comparable with other treatment regimens. This benefit amounted to an overall 15\% reduction in mortality at 30 days compared with the combined streptokinase groups. Initial subgroup analyses of the data identified certain baseline characteristics that seemed to predictf greater or lesser treatment benefit, but controversy has arisen concerning the interpretation of these analyses ${ }^{2}$ because sampling fluctuations affect all subgroup analyses.

The goal of this investigation was to develop a simplified statistical model that would allow the clinician to estimate the degree of expected benefit with 
accelerated TPA compared with streptokinase for individual patients.

\section{METHODS}

Patient population. The GUSTO-I trial randomly assigned 41,021 patients with suspected acute myocardial infarction and ST-segment elevation within 6 hours of the onset of acute ischemic symptoms. Patients with a history of stroke, bleeding diathesis, recent surgery or large-vessel puncture, or prior streptokinase or anistreplase therapy were excluded. Patients were not excluded on the basis of age or hemodynamic status alone. The details of the baseline characteristics of the population have been described. ${ }^{1}$

Medical therapy. The details of the protocol have also been described elsewhere. ${ }^{1}$ Patients were randomly assigned by telephone to one of four treatments: (1) streptokinase 1.5 million $U$ over a 1-hour period, with subcutaneous heparin 12,500 $\mathrm{U}$ twice daily beginning 4 hours after the start of thrombolytic therapy; (2) streptokinase 1.5 million $U$ over a 1-hour period, with an intravenous heparin bolus of 5,000 $\mathrm{U}$ then $1,000 \mathrm{U} / \mathrm{hr}$, with dose adjustment to maintain an activated partial thromboplastin time of 60 to 85 seconds; (3) accelerated TPA, consisting of a bolus of $15 \mathrm{mg}$ followed by an infusion of $0.75 \mathrm{mg} / \mathrm{kg}$ (up to $50 \mathrm{mg}$ ) over a 30 -minute period and $0.5 \mathrm{mg} / \mathrm{kg}$ (up to 35 $\mathrm{mg} / \mathrm{kg}$ ) over the next hour, accompanied by the same intravenous heparin regimen; or (4) the combination of intravenous TPA $(1.0 \mathrm{mg} / \mathrm{kg}$ over a 1 -hour period, not to exceed $90 \mathrm{mg}$, with one tenth given as a bolus) and streptokinase (1.0 million U over a 1 -hour period), given concurrently but through separate catheters, accompanied by the same intravenous heparin regimen. Aspirin was given to all patients at the time of randomization and daily thereafter, and intravenous $\beta$-blockade was recommended in all patients without contraindications. All other medical therapy was left to the discretion of the treating physician.

Data collection. Baseline characteristics reflecting demographics, cardiovascular history, and presentation status were collected by the telephone randomization system and on case record forms. The case record forms were reviewed at the Data Coordinating Center for completeness and consistency, and a series of computerized audit checks was completed on every form. All discrepancies were checked again through a query system in which the clinical sites were contacted to review the original record. A sample of $12 \%$ of the cases was reviewed in detail by independent monitors who compared the case report form with the medical record. Survival status was ascertained at 30 days in 40,830 (99.5\%) of the patients.

Mortality model. A statistical model was developed to examine the relation between baseline characteristics and 30-day survival. ${ }^{3}$ This model was a multivariable binary logistic model, ${ }^{4,5}$ for which the relations of continuous predictor variables with mortality were checked by cubic spline functions (piecewise polynomials) and appropriately characterized without assuming linearity. ${ }^{6-8}$ Interactions of key prognostic variables with treatment and interac- tions among the predictors other than treatment were examined to assess the additivity of these factors on the log odds of mortality. The final model included the following variables: age, systolic blood pressure, Killip class, heart rate, site of current infarction, prior infarction, the interaction of age with Killip class, height, time to treatment, diabetes, weight, smoking status, type of thrombolytic therapy, prior bypass surgery, hypertension, and cerebrovascular disease.

To apply this mortality model to predict the degree of treatment benefit, we let $\mathrm{X}_{1}, \mathrm{X}_{2}, \ldots, \mathrm{X}_{p}$ denote these risk factors (excluding treatment) and initially wrote the model as though each factor was represented by a single regression coefficient $(\beta)$ in the logistic model. Assume that only one of the factors $\left(\mathrm{X}_{p}\right)$ interacts with treatment and that the interaction is of a simple linear form. The basic logistic model formulation for the probability that a patient who has risk factor values $\mathrm{X}_{1}, \mathrm{X}_{2}, \ldots, \mathrm{X}_{p}$ and receives streptokinase will die within 30 days is Prob[Death $\mid \mathrm{SK}]=$

$$
\text { (1) } \frac{1}{1+\exp \left[-\left(\beta 0+\beta_{1} \mathrm{X}_{1}+\ldots+\beta_{p} \mathrm{X}_{p}\right)\right]}
$$

(where SK is streptokinase), and the probability of death for a patient who receives accelerated TPA is $\operatorname{Prob}[$ Death $\mid \mathrm{TPA}]=$

$$
\text { (2) } \left.\left.\frac{1}{1+\exp \left[-\left(\beta_{0}+\beta_{1} \mathrm{X}_{1}+\ldots+\beta_{p} \mathrm{X}_{p}\right.\right.}+\gamma+\delta \mathrm{X}_{p}\right)\right]
$$

In equation (2), $\gamma$ is the change in the log odds of death from TPA when $\mathrm{X}_{p}=0$, and $\delta$ is the additional change in the log odds from TPA per unit change in $\mathrm{X}_{p}$. These equations hold if the overall model holds and if $\mathrm{X}_{p}$ is the only risk factor that modifies the relative effect of treatment. The mortality difference or absolute treatment benefit is given by the difference in these two equations.

The mortality model above was applied to the GUSTO-I patient population and to selected subsets of the population to estimate differences in 30-day mortality rates for patients treated with streptokinase rather than with accelerated TPA. Because no significant interactions occurred between treatment and any of the other key prognostic factors $(\delta=0)$, the relative treatment effect of accelerated TPA versus streptokinase was characterized in terms of a single odds ratio $\left(e^{\gamma}\right)$, and the absolute difference in the probability of death for a patient treated with streptokinase versus that of a similar patient who received accelerated TPA was expressed as:

$$
\text { (3) } \Delta=P-\frac{P}{P+(1-P) \times \frac{1}{O R}}
$$

where $P$ denotes the probability of death for a patient treated with streptokinase and OR is the odds ratio 


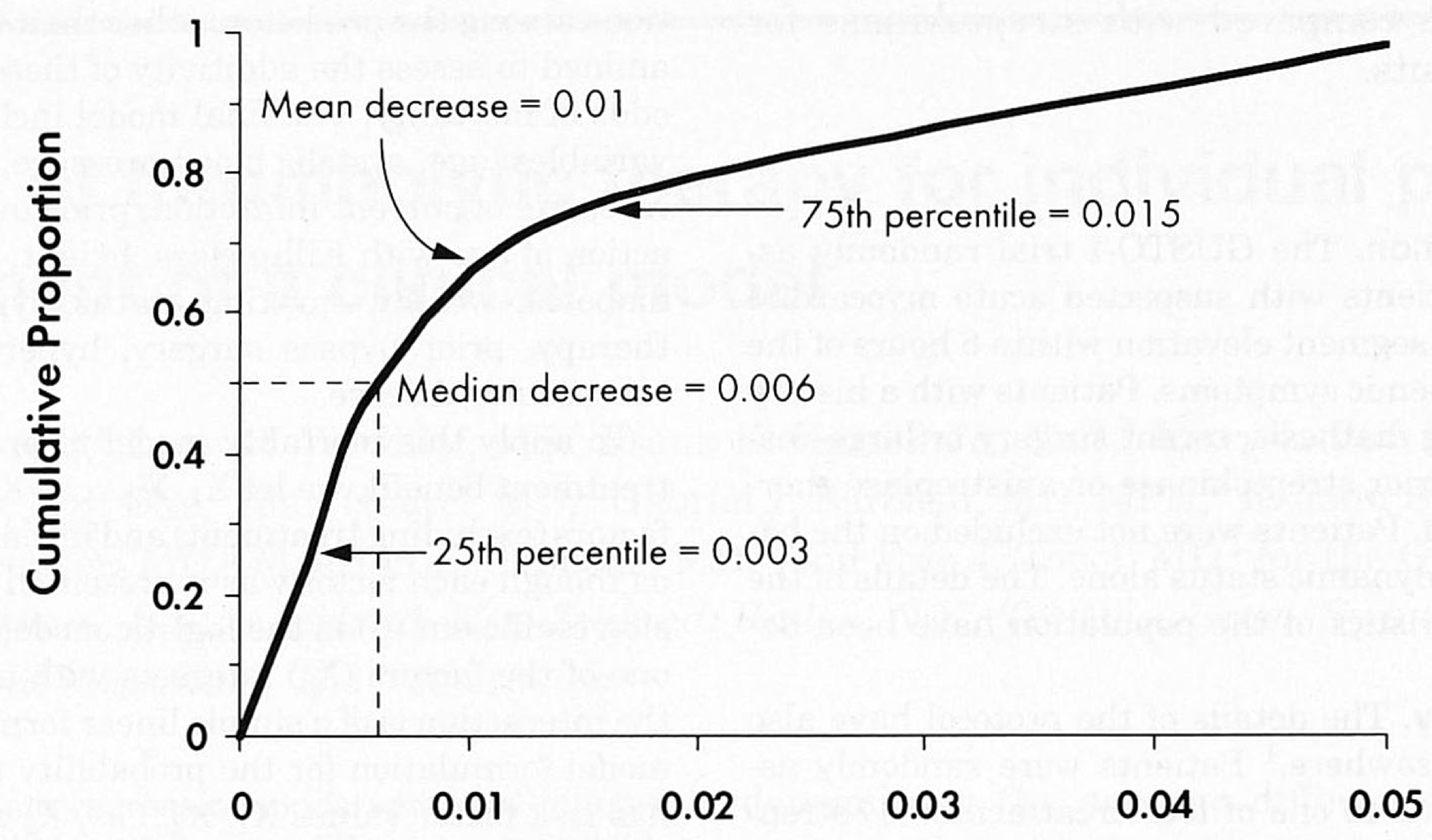

Absolute Decrease in Risk of Death

Fig. 1. Absolute mortality decrease with accelerated TPA treatment vs baseline mortality risk (assuming streptokinase treatment). Dashed line represents expected mortality as function of underlying patient risk, generated from statistical model developed from all available baseline characteristics [see equation (3)]. Dots reflect difference in proportions of patients who died in each decile of predicted risk for patients who received TPA versus streptokinase. Solid line represent nonparametric estimate of relation between predicted patient risk and observed mortality difference in TPA vs combined streptokinase groups. $S K$, Streptokinase.

or relative treatment effect of accelerated TPA compared with streptokinase. The background risk level $P$ has a dominating effect on the mortality difference. On the basis of this relation, the change in the probability of death expected from treatment with accelerated TPA compared with streptokinase was calculated for each patient in the GUSTO-I population, and the distribution of this change in risk was characterized with a cumulative distribution function. Also, the relation between baseline risk $P$ (with streptokinase treatment) and absolute mortality reduction with accelerated TPA as described by equation (3) is depicted as the "theoretical" curve in Fig. 1.

To demonstrate that treatment-specific mortality differences estimated from this model were consistent with observed differences, we used two approaches. First, we divided the population who received either streptokinase monotherapy into deciles of underlying risk, as predicted by equation (1), and similarly divided the patients who received accelerated TPA with the same intervals of risk for both treatment groups. Within each decile and treatment group the proportion of deaths was computed. We then calculated the difference between the two proportions within each risk decile and plotted for each decile (series "by decile" in Fig. 1). Second, we computed "lowess" nonparametric regression esti- mates (the "smoothed empirical" series in Fig. 1 ) $^{9}$ of the predicted risk versus the binary mortality indicator. This algorithm does not require construction of arbitrary risk intervals butinstead assumes a smooth relation between predicted risk and actual mortality; lowess does not assume any mathematic form for this relation. Lowess uses a moving least-squares linear regression fit on all data points $(\hat{\mathrm{P}}, \mathrm{Y})$ for each treatment group, where $\hat{P}$ denotes the predicted probability of death and $\mathrm{Y}$ denotes the outcome status for one subject ( $0=$ alive, $1=$ dead). Without making assumptions, the lowess regression estimates can easily be used to derive a nonparametric estimate of the relation between $\hat{\mathrm{P}}_{\mathrm{SK}}$ and $\mathrm{P}_{\mathrm{SK}}-\mathrm{P}_{\mathrm{TPA}}$, where $\hat{\mathrm{P}}_{\mathrm{SK}}$ is the predicted mortality for a patient who receives streptokinase, $\mathrm{P}_{\mathrm{SK}}$ is the actual mortality for a patient who received streptokinase, and $\mathrm{P}_{\text {TPA }}$ is the actual mortality for a patient treated with TPA. The lowess estimate from $\left(\hat{\mathrm{P}}_{\mathrm{SK}}, \mathrm{Y}\right)$ for all patients who received streptokinase is evaluated at $\hat{\mathrm{P}}_{\mathrm{SK}}=0$, $0.0035,0.007,0.0105, \ldots, 0.35$. Then the lowess estimate from $\left(\hat{\mathrm{P}}_{\mathrm{SK}}, \mathrm{Y}\right)$ for all patients who received TPA is evaluated at the same sequence of $\hat{P}_{S K}$ values. At each $\hat{\mathrm{P}}_{\mathrm{SK}}$ point, the two lowess estimates are subtracted to estimate the difference in the probabilities of death.

Development of a simple model. To obtain a simplified risk model that would permit easier manual computation 
of the differences in mortality rate expected between streptokinase and accelerated TPA, we performed a backward stepdown variable selection from the full mortality model based on the sample of GUSTO-I patients that excluded the TPA combination therapy, stopping when the next variable to be deleted (adjusted for the other variables remaining in the model) had a $\chi^{2}$ statistic $>90$ (the cutoff of 90 was chosen because there was a sizable break in the $\chi^{2}$ values at that point). The resulting model had the following variables and transformations that met this criterion: age, systolic blood pressure, Killip class, heart rate, site of current infarction, and prior myocardial infarction. A significant age-by-Killip class interaction was included in the model (even though its $\chi^{2}$ statistic was $<90$ ) because age and Killip class were such important predictors. Type of thrombolytic therapy was also retained in the simplified model. Table I shows the importance of each variable in the reduced model. Although the number of variables was considerably reduced, $>90 \%$ of the total prognostic information was retained. ${ }^{3}$ From this reduced model, we developed a nomogram to allow rapid calculation of the predicted absolute mortality reduction with accelerated TPA treatment compared with streptokinase treatment. Because the mortality in the two streptokinase monotherapy arms was essentially identical, the two regression coefficients associated with these strategies were averaged when predicting the probability of death with streptokinase. The nomogram was constructed with the "nomogram" function in the "design" library of UNIX S-PLUS statistical functions, which is available electronically in the public domain. ${ }^{10}$

Mortality has been expressed in this report in terms of proportions (i.e., on a probability scale) rather than as percentages.

\section{RESULTS}

Fig. 1 shows the relations between the underlying risk of death with streptokinase therapy and the absolute reduction in mortality with accelerated TPA predicted by the model, for deciles of patient risk, and by using empirical data. Patients who gained the most from accelerated TPA therapy were those with the highest predicted underlying risk. In addition, the predicted treatment benefits (theoretical curve) closely matched the observed benefits (deciles). The smoothed nonparametric estimates (smoothed empirical), which are less variable than the decile estimates because of the use of predicted mortality as a continuous variable, also validate model estimates of mortality differences.

Table II provides the observed mortality rate reduction with TPA versus that expected from the model prediction for selected patient subgroups. Observed mortality rate reductions are differences in subgroup proportions, whereas expected reductions are means of differences projected from equation (3)
Table I. Variables predictive of 30-day mortality in reduced GUSTO-I model

\begin{tabular}{lc}
\hline \multicolumn{1}{c}{ Variable } & Adjusted $\chi^{2 *}$ \\
\hline Age (yrs) & $1390(4 \mathrm{df})$ \\
Systolic blood pressure $(\mathrm{mm} \mathrm{Hg})$ & 533 \\
Killip class & $428(6 \mathrm{df})$ \\
Heart rate (per min) & $325(2 \mathrm{df})$ \\
Location of infarction & $147(2 \mathrm{df})$ \\
Previous myocardial infarction & 92 \\
Age by Killip class interaction & $31(3 \mathrm{df})$ \\
All thrombolytic treatments & $15.5(3 \mathrm{df})$ \\
\hline
\end{tabular}

$d f$, Degrees of freedom.

*Indicates independent contribution of each variable after adjusting for all other factors in list.

for the same subgroup. Again, high-risk patients derived the greatest benefit from TPA treatment, and there was a good overall match between the predicted and observed benefits. The one exception to the accurate prediction of outcome in subgroups by the model was the small group of elderly patients in cardiogenic shock, in whom the benefit was less than that predicted.

The distribution of the expected absolute survival benefit with accelerated TPA versus streptokinase for the entire GUSTO-I population is shown in Fig. 2. The median predicted survival benefit was 0.006 compared with a mean predicted benefit of 0.01 . The distribution of expected benefit as a function of underlying risk encompassed a broad spectrum: one fourth of the patients showed an expected survival benefit of $<0.003$, and another quarter had an expected benefit of $>0.015$.

Because of the low underlying mortality risk in patients younger than age 50, the distribution was skewed toward a lower benefit in these patients (data not shown). Table III illustrates the application of this information, which provides expected outcomes for four representative patients. For patients $\mathrm{A}$ and $\mathrm{B}$, despite having the same (young) age, the expected mortality rates and the treatment benefit of accelerated TPA were markedly different. Similarly, although the risk of death for patients $\mathrm{C}$ and $\mathrm{D}$ was much higher because of their increased age, a substantial difference in expected treatment benefit was evident.

Table IV shows the same type of comparison as a function of time from symptom onset to treatment. Because many of the patients treated late had a number of other high-risk characteristics, time to treatment makes relatively little contribution to treatment effect compared with other characteristics representing the underlying severity of illness.

Entry blood pressure had no substantial effect on 


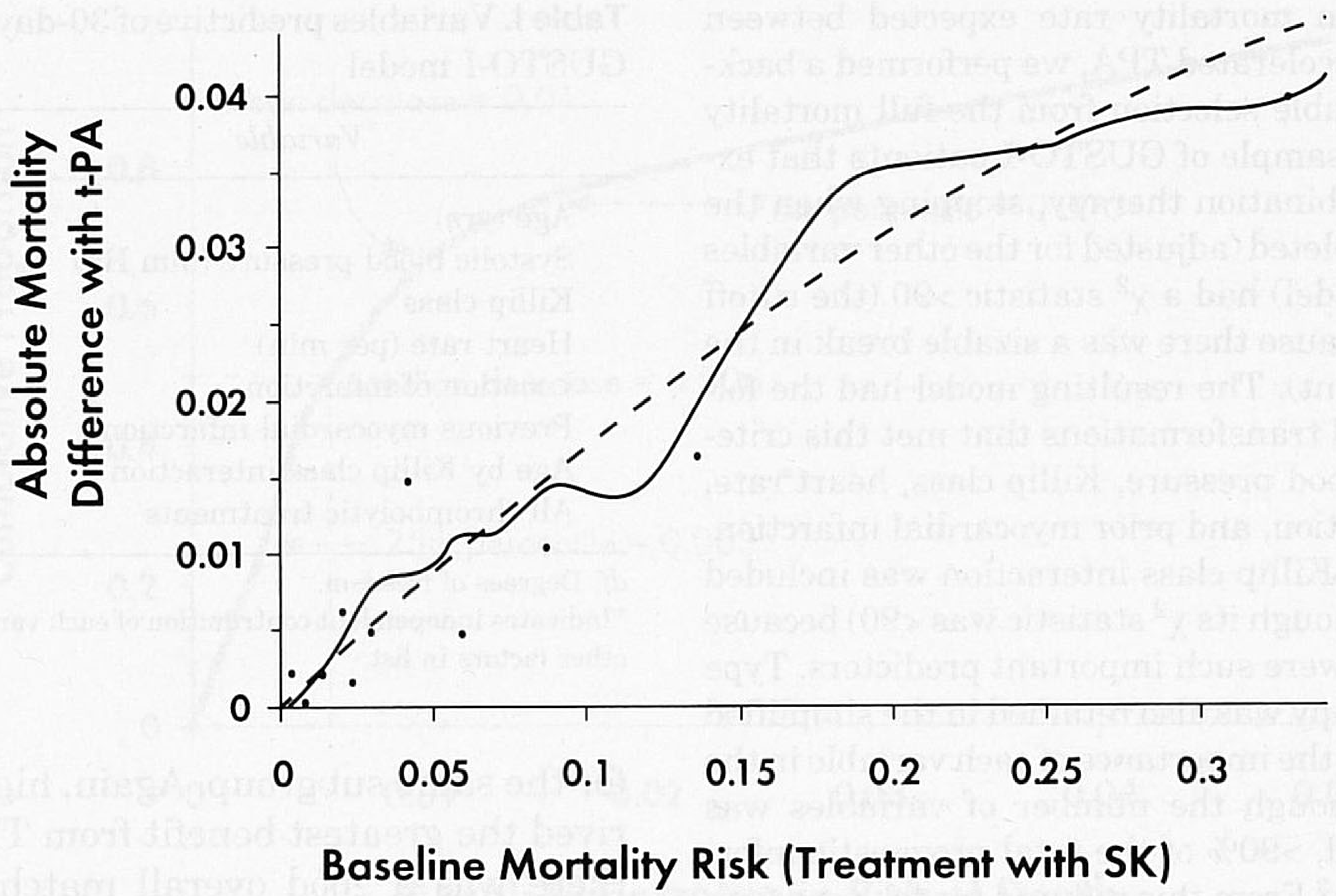

Fig. 2. Cumulative distribution of benefit with accelerated TPA treatment vs streptokinase treatment for entire GUSTO-I population. SK, Streptokinase.

Table II. Predicted vs observed mortality decrease with accelerated TPA treatment in selected patient subgroups

\begin{tabular}{lrrr}
\hline & & \multicolumn{2}{c}{ Absolute mortality decrease } \\
\cline { 4 - 4 } & $n$ & Predicted & Observed \\
\hline Killip classes I-II & & & 0.008 \\
Age $<75$ yrs & 35,166 & 0.027 & 0.0198 \\
Age $\geq 75$ yrs & 4800 & 0.038 & 0.037 \\
Killip classes III-IV & & 0.04 & 0.009 \\
Age $<75$ yrs & 629 & & 0.0104 \\
Age $\geq 75$ yrs & 235 & 0.0086 & 0.0088 \\
Time to treatment (hr) & 8420 & 0.0107 & 0.0126 \\
$0-2$ & 15,070 & 0.0142 & 0.0134 \\
$2-4$ & 5810 & 0.0146 & 0.0178 \\
$4-6$ & 1347 & & 0.0051
\end{tabular}

the expected benefit of accelerated TPA except in patients with hypotension, who could expect greater benefit. As demonstrated with representative individual patients in Table $\mathrm{V}$, a greater treatment benefit is seen in patients with lower blood pressure, although patient $\mathrm{D}$, who was in hemodynamic distress despite a good blood pressure, had a high mortality risk and a large expected treatment benefit.

When the location of myocardial infarction was examined, patients with anterior infarction had the greatest expected benefit, but many patients with inferior infarction also showed a substantial survival advantage. Table VI demonstrates that even though infarct location is important in stratifying expected treatment benefit, considering this characteristic in isolation can lead to inaccurate choices. Patient B with an inferior infarction would have a substantially greater expected benefit with accelerated TPA than patient $\mathrm{C}$ with an anterior infarction because patient $\mathrm{C}$ was hemodynamically stable.

Fig. 3 shows the nomogram developed from the reduced mortality model. By assessing a point score for each predictive variable and determining a point total for a given patient, a clinician can quickly estimate the expected mortality risk if the patient receives streptokinase. In addition, the absolute reduction in risk if accelerated TPA is substituted can easily be obtained. 
Table III. Expected outcomes for representative patients: Age

\begin{tabular}{|c|c|c|c|c|}
\hline & Patient A & Patient B & Patient $C$ & Patient $D$ \\
\hline Age & 50 & 50 & 75 & 75 \\
\hline Systolic BP (mm Hg) & 160 & 95 & 160 & 95 \\
\hline Killip class & I & $>\mathrm{I}$ & I & $>\mathrm{I}$ \\
\hline Location of infarction & Inferior & Anterior & Inferior & Anterior \\
\hline \multicolumn{5}{|c|}{ Expected 30 -day mortality rate } \\
\hline Accelerated TPA & 0.008 & 0.131 & 0.045 & 0.330 \\
\hline Streptokinase & 0.01 & 0.156 & 0.054 & 0.377 \\
\hline No. patients in GUSTO-I* & 1377 & 143 & 1232 & 247 \\
\hline
\end{tabular}

$B P$, Blood pressure.

*Number of patients within 10 points for continuous measures and within same discrete categories.

Table IV. Expected outcomes for representative patients: Time to treatment

\begin{tabular}{|c|c|c|c|c|}
\hline & Patient A & Patient B & Patient $C$ & Patient $D$ \\
\hline Time to treatment & $1 \mathrm{hr}$ & $1 \mathrm{hr}$ & $5 \mathrm{hr}$ & $5 \mathrm{hr}$ \\
\hline Age & 50 & 75 & 50 & 75 \\
\hline Systolic BP (mm Hg) & 160 & 95 & 160 & 95 \\
\hline Killip class & I & $>\mathrm{I}$ & I & $>\mathrm{I}$ \\
\hline Location of infarction & Inferior & Anterior & Inferior & Anterior \\
\hline \multicolumn{5}{|c|}{ Expected 30-day mortality rate } \\
\hline Accelerated TPA & 0.006 & 0.31 & 0.010 & 0.36 \\
\hline Streptokinase & 0.008 & 0.35 & 0.012 & 0.41 \\
\hline No. patients in GUSTO-I* & 381 & 53 & 258 & 66 \\
\hline
\end{tabular}

$B P$, Blood pressure.

* Number of patients within 10 points for continuous measures, within 1 hour of time point, and within same discrete categories.

Table V. Expected outcomes for representative patients: Systolic blood pressure

\begin{tabular}{|c|c|c|c|c|}
\hline & Patient A & Patient $B$ & Patient $C$ & Patient $D$ \\
\hline Age & 50 & 75 & 50 & 75 \\
\hline Systolic BP $(\mathrm{mm} \mathrm{Hg})$ & 95 & 95 & 160 & 160 \\
\hline Killip class & I & $>\mathrm{I}$ & I & $>\mathrm{I}$ \\
\hline Location of infarction & Inferior & Anterior & Inferior & Anterior \\
\hline \multicolumn{5}{|c|}{ Expected 30 -day mortality rate } \\
\hline Accelerated TPA & 0.016 & 0.33 & 0.008 & 0.17 \\
\hline Streptokinase & 0.020 & 0.38 & 0.010 & 0.20 \\
\hline No. patients in GUSTO-I* & 1269 & 247 & 1377 & 330 \\
\hline
\end{tabular}

$B P$, blood pressure.

*Number of patients within 10 points for continuous measures and within same discrete categories.

\section{DISCUSSION}

Therapeutic decision-making in an emergency situation requires considerable knowledge yet permits little time for reflection or consultation. The choice of thrombolytic agent provides a particularly fertile paradigm for the problem of emergency decisionmaking because of the large amount of data available and the importance of minimizing the time until the decision is made. Additionally, as the biologic basis for disease continues to be unraveled, clinicians will increasingly need to choose among multiple effective therapies. The major finding of our analysis was that although the relative benefit of accelerated TPA is constant, the absolute benefit is a function of the un- derlying risk of the patient, and no powerful biologic modifiers of this general effect were apparent.

These general results are similar to the findings of others who have used quantitative methods to estimate benefit of different thrombolytic therapy regimens. Simoons and Arnold ${ }^{11}$ developed a simple risk score based on age, sex, previous myocardial infarction, treatment delay, and electrocardiographic characteristics and similarly concluded that the degree of benefit of thrombolytic therapy and of more effective thrombolytic regimens was directly related to the extent of underlying mortality risk. A more detailed analysis, including estimates of life expectancy, has extended this observation with much the same find- 
Table VI. Expected outcomes for representative patients: Location of infarction

\begin{tabular}{lcccc}
\hline & Patient A & Patient B & Patient C & Patient D \\
\hline Age & 50 & 75 & 50 & 75 \\
Systolic BP (mm Hg) & 160 & 95 & 160 & 95 \\
Killip class & I & $>$ I & I & Anterior \\
Location of infarction & Inferior & Inferior & & Anterior \\
Expected 30-day mortality & & 0.19 & 0.15 & 0.18 \\
$\quad$ Accelerated TPA & 0.010 & 0.22 & 1003 & 0.33 \\
$\quad$ Streptokinase & 1377 & 261 & 247 \\
No. patients in GUSTO-I* & & & \\
\hline
\end{tabular}

$B P$, blood pressure.

*Number of patients within 10 points for continuous measures and within same discrete categories.

ings: accelerated TPA provides particular advantages for patients with combinations of high-risk characteristics. ${ }^{12}$ Our study extends these findings by providing empirical evidence from specific individual patient characteristics from a large randomized trial. For the purposes of illustration, this analysis combined the patients who were randomly assigned to receive streptokinase and either intravenous or subcutaneous heparin. This approach was taken because the protocol prospectively called for these groups to be combined if no significant difference in 30-day mortality was observed. In fact, the mortality at 30 days was quite similar $(0.073$ with streptokinase and subcutaneous heparin versus 0.074 with streptokinase and intravenous heparin); at 1 year of follow-up the mortality rates in the two groups were identical (0.10). ${ }^{13}$ Despite the lack of significant difference between the two regimens in terms of 30-day or 1-year mortality, a separate comparison of 30-day mortality with streptokinase and subcutaneous heparin subtracting 0.001 from the tables and figures does not change the basic message.

Clinical decision-making as traditionally taught in medical practice reflects a cognitive process referred to as heuristics, or rules of thumb. ${ }^{14}$ With this method, clinicians are taught to focus on individual patient characteristics (age, time to treatment, location of infarction) to allow the placement of patients into categories or subgroups. Although this thought process can lead to better decisions than broad application of therapies to entire diagnostic categories of patients, it falls short because multiple patient characteristics act jointly to influence both outcome and treatment effect. This finding is not unique to the treatment of acute myocardial infarction; the dominance of underlying risk as a determinant of absolute treatment benefit (number of lives saved) has been documented in many other disease states. In clinical trials of $\beta$-blocking agents in acute myocardial infarction, Yusuf et al. ${ }^{15}$ documented the same phenomenon. We have reported a similar find- ing among candidates for coronary artery bypass grafting, ${ }^{16}$ although the extent of coronary artery disease plays an important modifying role in terms of qualitative and quantitative interactions with the treatment. Yusuf et al. ${ }^{17}$ recently demonstrated this same concept in an overview of clinical trials of bypass surgery. The importance of this concept in the treatment of sepsis with new biologic interventions has been emphasized. ${ }^{18}$

In GUSTO-I, treatment with accelerated TPA and intravenous heparin led to a $15 \%$ relative reduction in the odds of death compared with streptokinase (odds ratio of 0.85 ). The application of these findings has been complicated by the cost issue many practitioners, hospitals, and health care systems have tried to identify patients who receive the most benefit so that the more expensive treatment might be reserved for them. ${ }^{19}$ This effort has been supported by pathophysiologic reasoning, particularly regarding the four characteristics chosen as examples in this study: age, time to treatment, infarct location, and systolic blood pressure. However, the underlying mortality risk of the patient was far more important than any of these factors individually.

One concern is that an aggressive therapy may actually be detrimental to patients at low risk of death because most potent therapies have a finite risk of catastrophic outcomes. In the treatment of sepsis, this risk has been quantified. ${ }^{18}$ Similarly, in patients with minimal anatomic disease, an excess risk with coronary artery bypass grafting has been documented. ${ }^{20}$ In the case of accelerated TPA, the excess risk of intracranial hemorrhage is a concern. However, because younger patients have a very low risk of intracranial hemorrhage, the treatment effect was not reversed, even in the lowest-risk group. Similar findings have been reported by Boersma et al., ${ }^{12}$ who used an overview of available evidence. In a hypothetical decision-analysis construct, Hillegass et al. ${ }^{21}$ found little impact from intracranial hemorrhage 


\section{Find Points for Each Marker}

\begin{tabular}{|c|c|c|c|c|c|c|c|c|}
\hline \multicolumn{2}{|c|}{ Killip Class I } & \multirow{2}{*}{$\begin{array}{l}\text { II } \\
\text { Points }\end{array}$} & \multirow{2}{*}{$\begin{array}{l}\text { III } \\
\text { Points }\end{array}$} & \multirow{2}{*}{$\begin{array}{l}\text { IV } \\
\text { Points }\end{array}$} & \multicolumn{2}{|c|}{ Heart Rate } & \multirow{2}{*}{$\begin{array}{l}\text { Systolic BP } \\
40\end{array}$} & \multirow{2}{*}{$\begin{array}{l}\text { Points } \\
34\end{array}$} \\
\hline Age & Points & & & & Per min & Points & & \\
\hline 30 & 19 & 35 & 48 & 53 & 0 & 12 & 60 & 25 \\
\hline 40 & 28 & 42 & 53 & 59 & 10 & 10 & 80 & 17 \\
\hline 50 & 38 & 49 & 59 & 65 & 20 & 7 & 100 & 8 \\
\hline 60 & 47 & 56 & 64 & 70 & 30 & 5 & $120+$ & 0 \\
\hline 70 & 57 & 63 & 70 & 76 & 40 & 2 & & \\
\hline 80 & 66 & 70 & 75 & 82 & 50 & 0 & Prior MI & Point \\
\hline 90 & 75 & 77 & 81 & 88 & 70 & 4 & Yes & 5 \\
\hline 100 & 85 & 84 & 86 & 94 & 90 & 8 & No & 0 \\
\hline \multirow[t]{6}{*}{110} & 94 & 91 & 92 & 100 & 110 & $\begin{array}{l}13 \\
17\end{array}$ & MI Location & Point \\
\hline & & & & & $\begin{array}{l}130 \\
150\end{array}$ & $\begin{array}{l}17 \\
21\end{array}$ & Anterior & \\
\hline & & & & & 170 & 25 & Inferior & 0 \\
\hline & & & & & 190 & 30 & Other & 3 \\
\hline & & & & & 210 & 34 & & \\
\hline & & & & & 230 & 38 & & \\
\hline
\end{tabular}

\section{Sum Points For All Risk Factors}

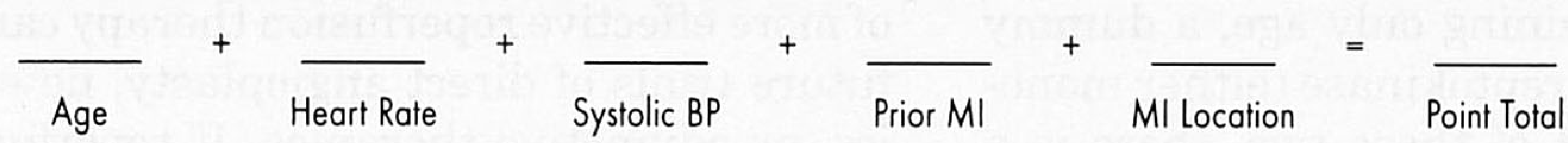

\section{Look Up Risk Corresponding to Point Total}

$\begin{array}{llll}\text { Points } & \text { SK Mortality } & \text { t-PA Mortality } & \text { t-PA Reduction } \\ 20 & 0.1 \% & 0.1 \% & - \\ 30 & 0.4 \% & 0.4 \% & - \\ 40 & 0.8 \% & 0.8 \% & 0.01 \% \\ 50 & 1.7 \% & 1.4 \% & 0.3 \% \\ 60 & 3.5 \% & 2.8 \% & 0.8 \% \\ 70 & 10 \% & 8.3 \% & 1.7 \% \\ 80 & 20 \% & 17 \% & 3 \% \\ 90 & 40 \% & 35 \% & 5 \%\end{array}$

Fig. 3. Nomogram to determine estimated 30-day mortality risk with streptokinase (SK) treatment for patients with given characteristics and absolute mortality reduction that would result from substitution of accelerated treatment. Point scores for each variable (1.) are added to produce overall point total (2.), which corresponds to mortality risk at bottom of figure (3.). (Note: Because age interacts with Killip class, age score that corresponds to patient's Killip class should be used.) $B P$, Blood pressure; $M I$, myocardial infarction.

differences (within the range found in GUSTO-I) on the choice of therapy.

Time to treatment is perhaps the most interesting pathophysiologic construct from the perspective of treatment benefit. Because the premise of the GUSTO-I trial was that rapid and sustained reperfusion would improve survival through salvage of more ischemic (but noninfarcted) myocardium, the concept that TPA would be differentially more effective in patients treated earlier makes pathophysiologic sense. Indeed, some commentators have sug- gested that streptokinase would be equally beneficial beyond 4 hours from symptom onset. ${ }^{22}$ However, the impact of time from symptom onset to treatment on the effect of treatment was modest at best. Indeed, the formal test for treatment interaction with time to treatment was not statistically significant. ${ }^{3}$ Because our statistical model does not reflect such an interaction, and because patients who are treated later are at somewhat higher risk (they are older and have other baseline risk factors), the model predicts slightly more treatment benefit in patients treated 
later. When viewed without taking these other factors into account, however, the empirical data show a nonsignificant trend in the other direction. ${ }^{23}$ The lack of a strong relation in empirical outcomes between time to treatment and benefit of accelerated TPA may reflect the complexity of estimating the time and constancy of artery occlusion in patients with varying sensitivities to ischemic pain. Many patients treated later probably either did not have total occlusion at the time of symptom onset or had intermittent occlusion. Alternatively, a small, incremental benefit may be gained even long after occlusion, especially in patients with substantial collateral flow.

Even in a study as large as GUSTO-I there is limited statistical power to detect a quantitative interaction between a risk factor and the relative benefit of accelerated TPA, mainly because of the low event rates. As an example, consider a formal test of whether the relative benefit depends on age, and assume that the age-benefit relation is linear. If we fit a model on patients containing only age, a dummy variable for TPA versus streptokinase (either monotherapy), and the product of these two, there is a slight difference in slope that indicates that TPA may be relatively less beneficial for older patients (with intersection of the two lines at about 100 years). This TPA-by-age interaction is not significant $(P=0.23)$. The estimates of the two age slopes are 0.008 and 0.0086 . The difference in slopes would need to be 0.014 instead of 0.0006 to provide 0.8 power to detect a significant differential effect of TPA according to age. No trial would likely enroll sufficient elderly patients to conduct this test with a high degree of confidence.

A clinician wishing to choose between accelerated TPA and streptokinase for treatment of a patient with ST-segment elevation infarction could use either the previously published regression coefficients ${ }^{3}$ or the nomogram in this article to make an individual determination. The regression model is more accurate for individual predictions, but the nomogram has the advantage of being easier to use. General knowledge that patients who are older or who have anterior infarction, hypotension, a worse Killip Class, or increased heart rate have higher mortality rate and therefore receive more benefit with TPA can provide nonquantitative guidance in treatment selection.

Similar hypotheses can be generated about other patient subgroups of interest. The increased stroke rate in older patients has led some to recommend streptokinase treatment for the elderly. ${ }^{24}$ However, our data clearly demonstrated a benefit of acceler- ated TPA in older patients equal to or greater than that in younger patients, except for possibly the very elderly. None of the other patient characteristics associated with increased risk of stroke (blood pressure, lower weight, female sex) were found to produce enough of an excess risk to override the mortality benefit of accelerated TPA. Although patients with anterior infarction generally achieved greater benefit than those with inferior infarction, the degree of benefit appeared most related to the contribution of infarct location to the patient's total risk and not to a specific biologic feature of anterior infarction.

The results of this study are relevant only to patients with ST-segment elevation and symptoms compatible with acute myocardial infarction. Although validating the findings in an independent population would be ideal, another large trial comparing accelerated TPA with streptokinase is unlikely. Nevertheless, the basic concept of the dominance of underlying risk in determining the benefit of more effective reperfusion therapy can be tested in future trials of direct angioplasty, new thrombolytics, or adjunctive therapies. If reperfusion therapy is to be rationed, then integration of multiple baseline characteristics to estimate underlying risk would be much more sensible than a strategy based merely on isolated individual characteristics of the patient.

\section{REFERENCES}

1. The GUSTO Investigators. An international randomized trial comparing four thrombolytic strategies for acute myocardial infarction. $\mathrm{N} \mathrm{Engl}$ J Med 1993;329:673-82.

2. Lee KL, Califf RM, Simes RJ, Van de Werf F, Topol EJ, for the GUSTO Investigators. Holding GUSTO up to the light. Ann Intern Med 1994; 120:876-81.

3. Lee KL, Woodlief LH, Topol EJ, Weaver WD, Betriu A, Col J, et al., for the GUSTO-I Investigators. Predictors of 30-day mortality in the era of reperfusion for acute myocardial infarction: results from an international trial of 41,021 patients. Circulation 1995;91:1659-68.

4. Cox DR. The regression analysis of binary sequences (with discussion). J R Statist Soc B 1958;20:215-42.

5. Walker SH, Duncan DB. Estimation of the probability of an event as a function of several independent variables. Biometrika 1967;54:16778.

6. Devlin TF, Weeks BJ. Spline functions for logistic regression modeling. Proceedings of the 11th Annual SAS Users Group International Conference 1986;646-51.

7. Harrell FE, Lee KL, Pollock BG. Regression models in clinical studies: determining relationships between predictors and response. J Natl Cancer Inst 1988;80:1198-202.

8. Smith PL. Splines as a useful and convenient statistical tool. Am Statistician 1979;33:57-62.

9. Cleveland WS. Robust locally weighted regression and smoothing scatterplots. J Amer Stat Assoc 1979;74:829-36.

10. Harrell, FE. Design: S functions for biostatistical/epidemiological modeling, testing, estimation, validation, graphics, prediction, and typesetting. Programs available from statlib@lib.stat.cmu.edu.

11. Simoons ML, Arnold AE. Tailored thrombolytic therapy. A perspective. Circulation 1993;88:2556-64. 
12. Boersma E, van der Vlugt MJ, Arnold AE, Deckers JW, Simoons ML. Estimated gain in life expectancy. A simple tool to select optimal reperfusion treatment in individual patients with evolving myocardial infarction. Eur Heart J 1996;17:64-75.

13. Califf RM, Topol EJ, Van de Werf F, Lee KL, Woodlief L, for the GUSTO Investigators [abstract]. One year followup from the GUSTO-I trial. Circulation 1994;90(Suppl I):I-325.

14. Krucoff MW, Croll MA, Pope JE, Granger CB, Pieper KS, Sigmon KN, et al. Heuristic and logistic principles of ST-segment interpretation in the time domain. Evolution in the context of the TAMI 7 trial design. J Electrocardiol 1990;23(Suppl I):6-10.

15. Yusuf S, Peto R, Lewis J, Collins R, Sleight P. Beta blockade during and after myocardial infarction: an overview of the randomized trials. Progr Cardiovasc Dis 1985;27:335-71.

16. Califf RM, Harrell FE Jr, Lee KL, Rankin JS, Hlatky MA, Mark DB, et al. The evolution of medical and surgical therapy for coronary artery disease. A 15-year perspective. JAMA 1989;261:2077-86.

17. Yusuf S, Zucker D, Peduzzi P, Fisher LD, Takaro T, Kennedy JW, et al. Effect of coronary artery bypass graft surgery on survival: overview of 10-year results from randomised trials by the Coronary Artery Bypass Graft Surgery Trialists Collaboration. Lancet 1994;344:563-70.
18. Knaus WA, Harrell FE, Fisher CJ, Wagner DP, Opal SM, Sadoff JC, et al. The clinical evaluation of new drugs for sepsis: a prospective study design based on survival analysis. JAMA 1993;270:1233-41.

19. Farkouh ME, Lang JD, Sackett DL. Thrombolytic agents: the science of the art of choosing the better treatment. Ann Intern Med 1994; $120: 886-8$.

20. Mark DB, Nelson CL, Califf RM, Harrell FE Jr, Lee KL, Jones RH, et al. The continuing evolution of therapy for coronary artery disease: initial results from the era of coronary angioplasty. Circulation 1994; 89:2015-25.

21. Hillegass WB, Jollis JG, Granger CB, Ohman EM, Califf RM, Mark DB. Intracranial hemorrhage risk and new thrombolytic therapies in acute myocardial infarction. Am J Cardiol 1994;73:444-9.

22. Fuster V. Coronary thrombolysis a perspective for the practicing physician. N Engl J Med 1993;329:723-5.

23. Topol EJ, Califf RM, Lee KL. More on the GUSTO trial. N Engl J Med 1993;331:277-8.

24. Krumholz HM, Pasternak RC, Weinstein MC, Friesinger GC, Ridker $\mathrm{PM}$, Tosteson AN, et al. Cost effectiveness of thrombolytic therapy in elderly patients with suspected acute myocardial infarction. N Engl J Med 1992;327:7-13

\section{BOUND VOLUMES AVAILABLE TO SUBSCRIBERS}

Bound volumes of the AMERICAN HEART JOURNAL are available only to subscribers for the 1997 issues from the Publisher at a cost of $\$ 92.50$ for domestic, $\$ 120.38$ for Canadian, and $\$ 112.50$ for international subscribers for Vol. 133 (January-June) and Vol. 134 (July-December). Shipping charges are included. Each bound volume contains a subject and author index, and all advertising is removed. Copies are shipped within 60 days after publication of the last issue in the volume. The binding is durable buckram with the journal name, volume number, and year stamped in gold on the spine. Payment must accompany all orders. Contact Mosby-Year Book, Inc., Subscription Services, 11830 Westline Industrial Dr., St. Louis, MO 63146, USA; phone (800)453-4351 or (314)4534351.

Subscriptions must be in force to qualify. Bound volumes are not available in place of a regular Journal subscription. 